\title{
Planejamento reprodutivo e a vulnerabilidade após o parto: uma coorte do sul do Brasil*
}

Reproductive planning and vulnerability after childbirth: a cohort from southern Brazil

Planeamiento reproductivo y la vulnerabilidad tras el parto: una cohorte del sur de Brasil

\author{
Márcia Aparecida dos Santos Silva CanarioI, Mariana Faria Gonçalves ${ }^{\text {II }}$, \\ Érica Mairene Bocate Teixeira ${ }^{\mathrm{III}}$, Áurea Fabrícia Amancio Quirino Silva ${ }^{\mathrm{IV}}$ \\ Rosângela Aparecida Pimenta Ferrariv , Sandra Marisa Pellosovi \\ Alexandrina Aparecida Maciel Cardelli ${ }^{\mathrm{vII}}$
}

\begin{abstract}
Resumo: Objetivo: analisar o planejamento reprodutivo no primeiro ano após o parto em mulheres que mantêm seguimento/acompanhamento em saúde na atenção primária e secundária. Método: trata-se de uma coorte prospectiva, conduzida com 300 mulheres, em município de grande porte do estado do Paraná, Brasil, entre julho de 2013 a março de 2015. Utilizou-se análise dos dados por meio do Teste Qui-quadrado. Resultados: foi verificada associação entre o uso de contraceptivo, situação conjugal e renda. A paridade e cesárea anterior também se associaram ao uso de contraceptivos. Verificou-se relevância estatística entre a gravidez planejada e estar grávida novamente. Houve associação entre inscrição no programa de planejamento reprodutivo e uso de contraceptivo, uso de método hormonal e acesso ao serviço de saúde. Ocorreu significância estatística entre a realização da revisão puerperal precoce ambulatorial e inscrição no planejamento reprodutivo. Conclusão: constatou-se restrição na aplicação das políticas de saúde sexual e reprodutiva após o parto.

Descritores: Anticoncepção; Atenção primária à saúde; Período pós-parto; Saúde sexual; Avaliação de programas e projetos de saúde
\end{abstract}

Abstract: Objective: it is to analyze the reproductive planning in the first year after the childbirth in women that keeps the healthy accompaniment in the primary and secondary attention. Method: it deals with a prospective cohort, which it

\footnotetext{
${ }^{\text {I }}$ Enfermeira. Mestre. Universidade Estadual de Londrina, Londrina, Paraná, Brasil. Email: marcia.s.s.canario@gmail.com, orcid.org/0000-0002-2882-6184

II Enfermeira. Mestre. Universidade Estadual de Londrina, Londrina, Paraná, Brasil. Email: mari.enfer@yahoo.com.br, orcid.org/0000-0002-5673-2556

III Enfermeira. Mestre. Universidade Estadual do Oeste do Paraná, Foz do Iguaçu, Paraná, Brasil. Email: ericamb13@hotmail.com, orcid.org/0000-0002-2774-2587

${ }^{\text {IV }}$ Enfermeira. Mestre. Universidade Estadual de Londrina, Londrina, Paraná, Brasil. Email: aureafabricia@hotmail.com, orcid.org/0000-0003-0454-3099

v Enfermeira. Doutora. Universidade Estadual de Londrina, Londrina, Paraná, Brasil. Email: ropimentaferrari@uel.br, orcid.org/0000-0003-0157-7461

${ }^{\mathrm{VI}}$ Enfermeira. Doutora. Universidade Estadual de Maringá, Maringá, Paraná, Brasil. Email: smpelloso@gmail.com, orcid.org/0000-0001-8455-6839

${ }^{\mathrm{VII}}$ Enfermeira. Doutora. Universidade Estadual de Londrina, Londrina, Paraná, Brasil. Email: macielalexandrina@gmail.com, orcid.org/0000-0002-0222-8821

* Extraído da tese "Planejamento reprodutivo no primeiro ano após o parto”, Programa de Pós-Graduação em Enfermagem, Universidade Estadual de Londrina, 2016.
} 
Planejamento reprodutivo e a vulnerabilidade após o parto: uma coorte do sul do Brasil | 2

was conducted with 300 women, in big towns of Parana State, Brazil, from July of 2013 to March of 2015. It was data used analysis through Qui- square tests. Results: it was verified association between contraceptive use, conjugal situation and earned income. The children's birth and Cesarean also are associated to the contraceptives usage. It was ascertained a statistics relevancy among the planned pregnancy with a woman being pregnant again. There was association between the registration in the health caring program and reproductive planning program, hormonal methods used in the health care access. It occurred a significantly statistics between the premature puerperal laboratory and inscription at the reproductive planning. Conclusion: it was verified the restriction in the implementation of sexual and reproductive health policies after the childbirth.

Descriptors: Contraception; Primary health care; Family; Postpartum period; Sexual health, Program evaluation

Resumen: Objetivo: evaluar el planeamiento reproductivo en el primer año tras el parto en mujeres que mantienen seguimiento/acompañamiento en salud en la atención básica y secundaria. Método: se trata de una cohorte prospectiva, que se realizó con 300 mujeres, en un gran municipio del estado de Paraná, Brasil, entre julio de 2013 y marzo de 2015. Se utilizó análisis de datos por medio de la Prueba Chi cuadrada. Resultados: se verificó asociación entre el uso de anticonceptivo, situación conyugal y renta. La paridad y cesárea anterior también se relacionaron al uso de anticonceptivos. Se constató relevancia estadística entre la gravidez planeada y estar embarazada nuevamente. Hubo asociación entre inscripción en el programa de planeamiento reproductivo y uso de anticonceptivo, uso de método hormonal y acceso al servicio de salud. Ocurrió significancia estadística entre realización de la revisión puerperal precoz en ambulatorio e inscripción en el planeamiento reproductivo. Conclusión: se constató restricción en la aplicación de las políticas de salud sexual y reproductiva tras el parto.

Descriptores: Anticoncepción; Atención primaria de salud; Periodo posparto; Salud sexual; Evaluación de programas y proyectos de salud

\section{Introdução}

O período após o parto é o momento em que as mulheres apresentam mudanças e adaptações físicas, emocionais e sociais, visando ao retorno da condição pré-gravídica. Entre as adaptações pelas quais a mulher passa está o retorno à prática sexual. ${ }^{1}$

A assistência à saúde reprodutiva é definida como o conjunto de métodos, técnicas, insumos e serviços que contribuem para a saúde e o bem-estar reprodutivo, prevenindo e resolvendo problemas de saúde reprodutiva. Assim, desde 2011, assistência em rede foi implementada para suprir as demandas do sistema, no intuito de enfrentar a fragilidade do acesso aos serviços de planejamento reprodutivo. ${ }^{2}$ As ações de planejamento reprodutivo são desenvolvidas, principalmente, pela Atenção Primária a Saúde (APS), sendo que a consulta puerperal é um momento oportuno para orientação e estímulo a essa prática. ${ }^{3} \mathrm{O}$ acesso à 
3 | Canario MASS, Gonçalves MF, Teixeira EMB, Silva AFAQS, Ferrari RAP, Pelloso SM, Cardelli AAM

contracepção é essencial para promover a saúde e autonomia das mulheres; um estudo de coorte prospectivo no Rio Grande do Sul verificou que $24,8 \%$ delas não realizaram consulta puerperal. ${ }^{4}$

Nesse sentido, o planejamento reprodutivo compreende a promoção da sexualidade saudável e segura, a regulação da fecundidade segundo o desejo do casal e preparação para uma paternidade responsável. ${ }^{5}$ No Brasil, a prestação do serviço de saúde no pós-parto não tem sido realizada de forma apropriada, sendo associada à baixa qualidade de assistência, pouca infraestrutura e desvalorização desse período. ${ }^{6}$

É oportuno destacar que o quinto objetivo do desenvolvimento sustentável é "alcançar a igualdade de gênero e empoderar todas as mulheres e meninas”. Dentre as ações programadas para esse objetivo está “Assegurar o Acesso Universal à Saúde Sexual e Reprodutiva e os Direitos Reprodutivos das Mulheres em Conformidade com o Programa de Ação da Conferência Internacional sobre População e Desenvolvimento e com a Plataforma de Ação de Pequim e os documentos resultantes de suas conferências de revisão". ${ }^{7}$ Portanto, nas últimas décadas a temática saúde reprodutiva têm despertado o interesse da comunidade cientifica e sua abordagem tem ido além do fator biológico, compreendendo as áreas éticas, políticas e sociais. A literatura demonstra que desfechos negativos da saúde materna e neonatal nos países em desenvolvimento poderiam ser evitados com o planejamento reprodutivo adequado. ${ }^{8}$

Por essa razão, este estudo tem por objetivo analisar o planejamento reprodutivo no primeiro ano após o parto em mulheres que mantêm seguimento/acompanhamento em saúde na atenção primária e secundária.

\section{Método}

Trata-se de uma coorte prospectiva conduzida com mulheres no pós-parto, residentes em município de grande porte do estado do Paraná, Brasil, seguidas nos níveis de atenção à saúde 
Planejamento reprodutivo e a vulnerabilidade após o parto: uma coorte do sul do Brasil | 4

primária e secundária. O cálculo da amostra foi realizado com base em 3.415 partos ocorridos no ano de 2012, sendo a amostra de 358 mulheres. Foram incluídas puérperas de risco obstétrico habitual ou intermediário, internadas nessa maternidade que residiam na zona urbana e que participaram das quatros etapas da pesquisa puerpério imediato, Revisão Puerperal Precoce Ambulatorial (RPPA), Revisão Puerperal Tardia (RPT), Revisão Puerperal Remoto (RPR); puérperas com diagnóstico de alto risco foram excluídas. O n final do estudo foi de 300 mulheres. A coleta de dados ocorreu no período de julho de 2013 a março de 2015, em quatro fases sequenciais.

A primeira fase teve início com a identificação diária das mulheres na unidade de alojamento conjunto em maternidade pública de risco habitual e intermediário, para obtenção de dados do Cartão da Gestante, prontuário e entrevista. Na segunda, procedeu-se à observação não participante da Revisão RPPA na maternidade (puerpério imediato, $7^{\circ}$ a $10^{\circ}$ dia após o parto). Na terceira, RPT (puerpério tardio, $42^{\circ}$ dia após o parto), e na quarta fase, RPR (puerpério remoto, um ano após o parto), realizaram-se visitas domiciliares.

Em 2011, a maternidade iniciou o serviço ambulatorial do puerpério, a princípio para reduzir os casos de infecção no pós-parto, as taxas de reinternação, o índice de mortalidade materna e manter um feedback da assistência prestada. Além disso, fornece informações sobre o aleitamento materno, captação de doação de leite e planejamento reprodutivo. O ambulatório atende de segunda a sextafeira das oito às treze horas, apenas puérperas cujo parto tenha sido realizado na própria instituição. Em média 12 mulheres comparecem ao dia para RPPA ( $1^{\circ}$ ao $10^{\circ}$ dia pós-parto). A equipe é composta por médico ginecologista, uma enfermeira obstetra e uma técnica em enfermagem. ${ }^{9}$

A RPPA é realizada diariamente pela enfermeira com auxílio da avaliação médica três dias na semana. Nos outros dias, caso exista necessidade, a avaliação é realizada pelo médico plantonista. O ambulatório possui um serviço de agendamento, sob responsabilidade da técnica de enfermagem. Um lembrete desse agendamento é anexado diariamente no cartão da criança, 
5 | Canario MASS, Gonçalves MF, Teixeira EMB, Silva AFAQS, Ferrari RAP, Pelloso SM, Cardelli AAM

vinte e quatro horas antes da alta hospitalar da díade. No caso de permanência do recémnascido na instituição, a puérpera não perde o direito ao atendimento ambulatorial. Para mulheres faltosas à revisão no dia agendado, existe a possibilidade de entrar em contato com a maternidade para reagendamento. No entanto, essa procura deve ocorrer no máximo em sete dias. O ambulatório não realiza a busca ativa das puérperas, devido a essa ação extrapolar o período preconizado pela instituição (puerpério imediato); porém, existe a contrarreferência de todas as puérperas para atenção primária no momento da alta hospitalar. ${ }^{9}$

A APS do município está organizada por meio da Estratégia de Saúde da Família (ESF), sendo dividida em 6 regiões que dispõem de 54 Unidade Básica de Saúde (UBS): 42 na zona urbana e 12 na rural. Além das equipes saúde da família, as UBSs da zona urbana contam com 10 equipes do Núcleo de Apoio à Saúde da Família (NASF), implantadas em 2008, constituídas por profissionais de diferentes áreas de saúde (nutrição, fisioterapia, farmácia, educação física, psicologia), que atuam junto às ESF em seus respectivos territórios. ${ }^{9}$

As ações de promoção em saúde, prevenção de agravos e visita domiciliar são comuns a todos membros da ESF e NASF. O enfermeiro, além de outras atribuições, possui atuação específica nas consultas de pré-natal e puerpério na UBS. Desde o início do pré-natal, o prontuário da gestante é separado e passa ser considerado prioridade assistencial. Após o parto, a funcionária responsável pelas ações técnico-administrativas da UBS verifica o nascimento da criança, por meio do sistema interno entre a maternidade e a UBS, e comunica ao enfermeiro responsável pela área de abrangência na qual reside a puérpera. O enfermeiro programa a RPP (entre o $7^{\circ}$ e $10^{\circ}$ dia pós-parto) realizada durante a visita domiciliar. Nesse atendimento, é realizado o agendamento da $\mathrm{RPT}$ (entre $30^{\circ}$ e $42^{\circ}$ dia pós-parto) a ser executada na UBS. No retorno da usuária à UBS, é realizado o agendamento para RPR (entre $43^{\circ}$ e $365^{\circ}$ pós-parto). ${ }^{9}$

O serviço de Planejamento Reprodutivo é disponibilizado a toda população do município, em idade fértil, que busque assistência sobre Planejamento Reprodutivo ou que seja 
Planejamento reprodutivo e a vulnerabilidade após o parto: uma coorte do sul do Brasil | 6

profissionalmente encaminhada para esta finalidade, preservando o conhecimento sobre os direitos sexuais e reprodutivos de homens, mulheres e casais. Em todas as Unidades de Saúde são disponibilizados métodos temporários: anticoncepcional oral e injetável (mensal e trimestral) e preservativo (condon masculino e feminino); Dispositivo Intrauterino (DIU) está disponível em algumas Unidades e na Unidade Municipal de Matriciamento e Ensino em Saúde da Família (UMMESF). Já para a realização dos métodos cirúrgicos (laqueadura e vasectomia) o município conta com as referências do Consórcio Intermunicipal de Saúde do Médio Paranapanema (CISMEPAR) e a Maternidade Municipal. ${ }^{9}$

Utilizou-se, na coleta de dados, instrumento com questões objetivas, como: aspectos sociodemográficos, planejamento reprodutivo (gravidez planejada, está gravida novamente, foi inscrita no programa de planejamento familiar, em uso de método contraceptivo, onde foi ofertado o método contraceptivo), revisão puerperal (puérpera retornou para revisão puerperal, quem realizou a consulta puerperal, orientações durante a consulta puerperal, realizada a contrarreferência, recebeu visita domiciliar (VD) de um profissional da UBS até o $7^{\text {o }}$ dia após o parto, por que procurou a UBS após o parto, agendaram a revisão puerperal tardia na UBS, qual profissional realizou o atendimento na revisão puerperal tardia, agendaram a revisão puerperal remota (após $43^{\circ}$ dia pós-parto) na UBS e qual profissional realizou o atendimento na revisão puerperal remota na UBS).

Testado previamente, após ajustes, iniciou-se a coleta até alcançar o $n$ amostral. Utilizou-se o programa Statistical Package for Social Sciences (SPSS), versão 20.0, para análise estatística por meio do Teste Qui-quadrado com nível de significância de 5\%, para verificar possíveis associações $(\mathrm{p}<0,05)$ com as variáveis dependentes: "uso método contraceptivo" e "inscrição no programa de planejamento reprodutivo". As variáveis independentes selecionadas contemplaram os aspectos sociais e demográficos, história obstétrica e planejamento reprodutivo. 
Em observância às determinações da Resolução 466/12, do Conselho Nacional de Saúde, o projeto de pesquisa foi aprovado pelo Comitê de Ética em Pesquisa Envolvendo Seres Humanos, sob o protocolo de aprovação CAAEE no 193525139.9.0000.5231, no dia 16 de julho de 2013. Após a aprovação, a instituição foi notificada para o início da coleta de dados em prontuários e antes da realização da entrevista com as mulheres/usuárias. Todas as participantes foram esclarecidas sobre os objetivos da pesquisa, a garantia do anonimato e o direito de participarem ou não, tendo o direito de manifestar sua aquiescência por meio da assinatura do Termo de Consentimento Livre e Esclarecido.

\section{Resultados}

A amostra do estudo foi caracterizada por mulheres jovens entre $20-34$ anos 69,6\% (209), com companheiro 85,6\% (257), renda de até um salário mínimo 69,6\% (209), sendo a maioria não remunerada $59,6 \%(179)$.

Houve diferença estatística entre a situação conjugal e o uso de método contraceptivo (MC) $(p<0,001)$ entre o $7^{\circ}$ e o $42^{\circ}$ dias após o parto. A maior proporção de mulheres que tinham companheiro fazia uso de contraceptivo quando comparadas às solteiras. O uso de MC também se associou significativamente com a renda no puerpério tardio: aquelas com renda mais baixa fizeram menor uso da contracepção. Ao engravidar, verificou-se que a maioria das mulheres não remuneradas não utilizavam o MC. Foi mais frequente o uso da contracepção entre as multíparas do que as primíparas.

A maior parte de mulheres com parto normal não fazia uso de contracepção. Na abordagem ao planejamento reprodutivo, identificou-se que houve relevância estatística entre a gravidez planejada e o uso de MC ao engravidar. A maior parte dos casais que não queriam engravidar estava utilizando contraceptivos. 
Planejamento reprodutivo e a vulnerabilidade após o parto: uma coorte do sul do Brasil | 8

Estar grávida novamente teve associação com uso de MC no puerpério remoto, em que 40\% das mulheres faziam uso de contraceptivo. O uso de MC também se associou significativamente ao motivo da mulher não estar inscrita no programa de planejamento reprodutivo (PPR) no puerpério tardio (Tabela 1$)$.

Tabela 1 - Uso de método contraceptivo. Londrina, PR, 2019

\begin{tabular}{|c|c|c|c|c|c|c|c|c|c|}
\hline \multirow{3}{*}{$\begin{array}{c}\text { Variáveis } \\
\text { Independentes }\end{array}$} & \multirow{2}{*}{\multicolumn{2}{|c|}{$\begin{array}{c}\text { Ao engravidar } \\
\text { Sim }\end{array}$}} & \multirow{3}{*}{$\begin{array}{l}\text { Valor } \\
\text { de p }\end{array}$} & \multirow{2}{*}{\multicolumn{2}{|c|}{$\begin{array}{l}7^{\circ}-42^{\circ} \text { dia } \\
\text { pós-parto } \\
\operatorname{Sim}\end{array}$}} & \multirow{3}{*}{$\begin{array}{l}\text { Valor } \\
\text { de } p\end{array}$} & \multirow{2}{*}{\multicolumn{2}{|c|}{$\begin{array}{c}1^{\circ} \text { ano após o } \\
\text { parto }\end{array}$}} & \multirow{3}{*}{$\begin{array}{c}\text { Valor } \\
\text { de } p\end{array}$} \\
\hline & & & & & & & & & \\
\hline & $\mathrm{n}$ & $(\%)$ & & $\mathrm{n}$ & $(\%)$ & & $\mathrm{n}$ & $(\%)$ & \\
\hline \multicolumn{10}{|l|}{ Situação conjugal } \\
\hline Com companheiro & 86 & 84,3 & & 225 & 90,4 & & 229 & 87,1 & \\
\hline Sem companheiro & 16 & 15,7 & 0,728 & 24 & 9,6 & $<0,001$ & 34 & 12,9 & 0,078 \\
\hline \multicolumn{10}{|l|}{ Renda } \\
\hline ×1 Salário Mínimo & 68 & 66,7 & & 167 & 67,1 & & 181 & 68,8 & \\
\hline 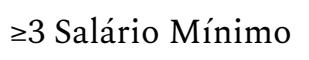 & 34 & 33,3 & 0,556 & 82 & 32,9 & 0,034 & 82 & 31,2 & 0,504 \\
\hline \multicolumn{10}{|l|}{ Ocupação } \\
\hline Remunerada & 51 & 50,0 & & 103 & 41,4 & & 109 & 41,4 & \\
\hline Não remunerada & 51 & 50,0 & 0,018 & 146 & 58,6 & 0,439 & 154 & 58,6 & 0,372 \\
\hline \multicolumn{10}{|l|}{ Paridade } \\
\hline Primíparas & 31 & 30,4 & & 90 & 36,1 & & 97 & 36,9 & \\
\hline Multíparas & 71 & 69,6 & 0,046 & 159 & 63,9 & 0,113 & 166 & 63,1 & 0,206 \\
\hline \multicolumn{10}{|l|}{ Tipo de parto } \\
\hline Normal & 76 & 74,5 & & 182 & 73,1 & & 191 & 72,6 & \\
\hline Cesárea & 26 & 25,5 & 0,96 & 67 & 26,9 & 0,379 & 72 & $27,4)$ & 0,055 \\
\hline \multicolumn{10}{|l|}{ Gravidez planejada } \\
\hline O casal queria & 43 & 42,2 & & 155 & 62,2 & & 158 & 60,1 & \\
\hline O casal não queria & 59 & 57,8 & $<0,001$ & 94 & 37,8 & 0,158 & 105 & 39,9 & 0,859 \\
\hline \multicolumn{10}{|l|}{ Grávida novamente* } \\
\hline $\operatorname{Sim}$ & - & - & & 1 & 52,2 & & 6 & 40,0 & $<0,001$ \\
\hline
\end{tabular}

* 15 mulheres engravidaram no período após o parto durante o estudo

A Tabela 2 mostra que a inscrição no PPR e o uso de MC apresentou associação positiva no puerpério precoce, tardio e remoto, demonstrando que a maior proporção de mulheres inscritas no programa fazia uso de contraceptivos. Da mesma forma, houve associação entre inscrição no PPR e o uso de método hormonal no puerpério precoce, tardio e remoto. O contraceptivo oral foi predominante no serviço de saúde no puerpério precoce, tardio e remoto. 
9 | Canario MASS, Gonçalves MF, Teixeira EMB, Silva AFAQS, Ferrari RAP, Pelloso SM, Cardelli AAM

No acompanhamento da revisão puerperal, verificou-se diferença estatística entre o agendamento na RPPA e a inscrição no PPR, em detrimento dos agendamentos na RPT e RPR. A maior proporção de mulheres que não tiveram o agendamento da consulta puerperal também não foi inscrita no PPR.

A maioria das mulheres não conhecia a existência do serviço de revisão puerperal. Entre as usuárias que realizaram a consulta puerperal, predominaram as orientações para cuidados com a criança.

Tabela 2 - Prática do planejamento reprodutivo. Londrina, PR, 2019

\begin{tabular}{|c|c|c|c|c|c|c|c|c|c|}
\hline \multirow{3}{*}{ Planejamento Reprodutivo } & \multicolumn{3}{|c|}{$10^{\circ}$ dia pós-parto } & \multicolumn{3}{|c|}{$42^{\circ}$ dia pós-parto } & \multicolumn{3}{|c|}{$1^{\circ}$ ano após o parto } \\
\hline & \multicolumn{2}{|c|}{$\operatorname{Sim}$} & \multirow{2}{*}{$\begin{array}{l}\text { Valor } \\
\text { de p }\end{array}$} & \multicolumn{2}{|c|}{ Sim } & \multirow{2}{*}{$\begin{array}{l}\text { Valor } \\
\text { de p }\end{array}$} & \multicolumn{2}{|c|}{$\operatorname{Sim}$} & \multirow{2}{*}{$\begin{array}{l}\text { Valor } \\
\text { de } \mathrm{p}\end{array}$} \\
\hline & $\mathbf{n}$ & $(\%)$ & & $\mathrm{n}$ & $(\%)$ & & $\mathrm{n}$ & $(\%)$ & \\
\hline \multicolumn{10}{|l|}{ Uso de método contraceptivo } \\
\hline Sim & 161 & 89,0 & 0,001 & 98 & 96,1 & $<0,001$ & 53 & 94 & 0,001 \\
\hline Não & 20 & 11,0 & & 4 & 3,9 & & 10 & 6,1 & \\
\hline \multicolumn{10}{|l|}{ Método contraceptivo } \\
\hline Barreira & 33 & 20,9 & 0,021 & 7 & 7,1 & $<0,001$ & 11 & 7,2 & 0,003 \\
\hline Hormonal & 117 & 70,9 & & 78 & 74,5 & & 109 & 27 & \\
\hline Cirúrgico & 13 & 8,2 & & 18 & 18,4 & & 33 & 21 & \\
\hline \multicolumn{10}{|l|}{ Local de oferta } \\
\hline Serviço de saúde & 164 & 85,1 & $<0,001$ & 92 & 90,2 & $<0,001$ & 127 & 83 & $<0,001$ \\
\hline Farmácia (compra) & 26 & 14,4 & & 7 & 6,9 & & 26 & 17 & \\
\hline Não Ofertado & 1 & 0,6 & & 3 & 2,9 & & - & - & \\
\hline \multicolumn{10}{|l|}{ Agendamento RP* } \\
\hline Sim & 27 & 33,3 & 0,002 & 27 & 49,1 & 0,488 & 59 & 36 & 0,394 \\
\hline Não & 54 & 66,7 & & 28 & 50,9 & & 104 & 64 & \\
\hline
\end{tabular}

${ }^{\star}$ Revisão puerperal

A Tabela 3 apresenta dados referentes ao acompanhamento da puérpera no ambulatório de revisão puerperal e na APS, apresentando significância estatística entre a realização da RPPA ao sétimo dia após o parto e inscrição no planejamento reprodutivo. A maior parte das mulheres que realizaram ao sétimo dia após o parto foram inscritas no PPR. Na RPP ao décimo dia após o parto, a maioria que estava inscrita no PPR foi atendida pelo enfermeiro. 
Planejamento reprodutivo e a vulnerabilidade após o parto: uma coorte do sul do Brasil | 10

Tabela 3 - Acompanhamento das puérperas pelo serviço de saúde. Londrina, PR, 2019

\begin{tabular}{|c|c|c|c|c|c|c|c|c|c|}
\hline \multirow{3}{*}{ Serviço de Saúde } & \multicolumn{3}{|c|}{$7^{70}$ dia pós-parto } & \multicolumn{3}{|c|}{$10^{\circ}$ dia pós-parto } & \multicolumn{3}{|c|}{$1^{\circ}$ ano após o parto } \\
\hline & \multicolumn{2}{|c|}{ Sim } & \multirow{2}{*}{$\begin{array}{l}\text { Valor } \\
\text { de } p\end{array}$} & \multicolumn{2}{|c|}{ Sim } & \multirow{2}{*}{$\begin{array}{l}\text { Valor } \\
\text { de p }\end{array}$} & \multicolumn{2}{|c|}{ Sim } & \multirow{2}{*}{$\begin{array}{l}\text { Valor } \\
\text { de } p\end{array}$} \\
\hline & $\mathrm{n}$ & (\%) & & $\mathrm{n}$ & (\%) & & $\mathrm{n}$ & (\%) & \\
\hline \multicolumn{10}{|l|}{ Revisão Puerperal } \\
\hline Sim & 55 & 39,3 & 0,087 & 181 & 96,3 & $<0,0001$ & 59 & 57,8 & 0,39 \\
\hline Não & 47 & 29,4 & & _- & _- & & 104 & 52,5 & \\
\hline \multicolumn{10}{|l|}{ Profissional } \\
\hline Médico & - & _ & 0,026 & 16 & 88,9 & 0,136 & 32 & 56,1 & 0,84 \\
\hline Enfermeiro & 37 & 48,7 & & 165 & 97,1 & & 27 & 60 & \\
\hline $\mathrm{ACS}^{*}$ & 5 & 20 & & & _ & & _- & _- & \\
\hline
\end{tabular}

*Agente Comunitário de Saúde

Houve associação, no puerpério tardio e remoto, entre procurar o serviço para planejamento reprodutivo e inscrição no PPR. A maior proporção que não buscou o serviço para planejamento reprodutivo no puerpério tardio também não foi inscrita no PPR. Porém, um ano após o parto, aquelas que procuraram o serviço para planejamento reprodutivo foram inscritas no PPR (Tabela 4).

Tabela 4 - Prática do planejamento reprodutivo após o parto. Londrina-PR, 2019

\begin{tabular}{|c|c|c|c|c|c|c|c|c|c|c|}
\hline \multirow{3}{*}{$\begin{array}{c}\text { Prática do } \\
\text { Planejamento } \\
\text { Reprodutivo }\end{array}$} & \multicolumn{5}{|c|}{$42^{\circ}$ dia pós-parto } & \multicolumn{5}{|c|}{1 ano pós-parto } \\
\hline & \multicolumn{2}{|c|}{ Sim } & \multicolumn{2}{|r|}{ Não } & \multirow{2}{*}{$\begin{array}{l}\text { Valor } \\
\text { de } p\end{array}$} & \multicolumn{2}{|c|}{ Sim } & \multicolumn{2}{|c|}{ Não } & \multirow{2}{*}{$\begin{array}{l}\text { Valor } \\
\text { de } p\end{array}$} \\
\hline & $\mathrm{n}$ & $\%)$ & $\mathrm{n}$ & $(\%)$ & & $\mathrm{n}$ & $(\%)$ & $\mathrm{n}$ & $(\%)$ & \\
\hline \multicolumn{11}{|l|}{ Buscou o serviço } \\
\hline Sim & 30 & 63 & 18 & 37,5 & & 102 & 93,6 & 7 & 6,4 & \\
\hline Não & 72 & 29 & 180 & 71,4 & $<0,001$ & 61 & 31,9 & 130 & 68,1 & $<0,001$ \\
\hline \multicolumn{11}{|l|}{ Ginecologista } \\
\hline Sim & 42 & 31 & 92 & 68,7 & & 83 & 54,6 & 69 & 45,4 & \\
\hline Não & 60 & 36 & 106 & 63,9 & 0,394 & 80 & 54,1 & 68 & 45,9 & 1,000 \\
\hline
\end{tabular}

\section{Discussão}

O conceito de planejamento familiar consta na Constituição Federal e é regulamentado pela Lei n 9.263/96, que compreende o planejamento familiar como um conjunto de ações de regulamentação da fecundidade, de forma a garantir direitos iguais, limitação ou aumento da prole pela mulher, pelo homem ou pelo casal. ${ }^{2}$ 
11 | Canario MASS, Gonçalves MF, Teixeira EMB, Silva AFAQS, Ferrari RAP, Pelloso SM, Cardelli AAM

Considerando a expansão do termo “planejamento familiar”, busca-se substituí-lo por “planejamento reprodutivo”, pois pode ser realizado de forma independente da instituição familiar. Firma-se no pressuposto da decisão livre e informada, dignidade humana e paternidade responsável, cabendo ao Estado a provisão de recursos para ação e manutenção dessa norma. ${ }^{10}$

O perfil encontrado neste estudo, de jovens adultas e adolescentes, corrobora com os dados da literatura, que identifica esta como a população que mais engravida no Brasil. ${ }^{11}$ Essa evidência acalora as discussões sobre os riscos aos quais essas jovens estão expostas, como baixo peso do recém-nascido, parto prematuro, parto operatório associado à laqueadura como medida de planejamento reprodutivo e gestações sucessivas com intervalo menor que dois anos. ${ }^{12}$

Outro questionamento é quanto às condições financeiras dessa população, pois o aumento da qualidade da saúde sexual reprodutiva influencia diretamente na redução da pobreza e melhoria das condições de vida dos indivíduos. ${ }^{11}$ Verificou-se que, no puerpério tardio, mulheres com recurso econômico menor ou precário não exerciam a prática de contracepção. Indivíduos com renda menor apresentam maiores necessidades e insatisfação com anticoncepção. Um estudo realizado na região do Oriente Médio de Gana identificou o aumento do uso de método contraceptivo conforme a paridade, para limitar o número de gestações. ${ }^{13}$

A literatura traz que a forma como o planejamento reprodutivo é desenvolvido na saúde pública não abrange o potencial do núcleo familiar, uma vez que são inexistentes quaisquer informações sobre como relacionar a renda com número de filhos, ou com investimentos na educação e na saúde de cada membro da família. ${ }^{6}$ O planejamento da vida reprodutiva consiste em incluir o que o indivíduo ou casal espera por seu futuro em relação ao ensino, ocupação e relacionamentos. Relatos femininos sobre uso de contraceptivos estão associados aos aspectos financeiros, profissão e educação. ${ }^{14}$

A anticoncepção e a existência de um parceiro mostraram-se influentes em todo o período do estudo, tendo maior adesão aos métodos contraceptivos as mulheres que conviviam 
Planejamento reprodutivo e a vulnerabilidade após o parto: uma coorte do sul do Brasil | 12

com companheiros. No entanto, houve uma tendência ao uso de métodos hormonais em comparação ao uso de métodos de barreira por essa população. Poucas mulheres faziam uso de preservativo, ficando vulneráveis às infecções sexualmente transmissíveis. Em uma pesquisa qualitativa sobre reprodução, práticas contraceptivas e abortamento voluntário, verificou-se que, à medida que ocorria estabilidade nos relacionamentos, os casais iam dispensando o uso do preservativo, subjugando os riscos. ${ }^{15}$

A autonomia sobre a fecundidade é vista como uma das amarras do processo de empoderamento feminino, recaindo, portanto, sobre a mulher as consequências de uma gestação não planejada. Assim, é compreendida como natural a sua busca solitária por contracepção. ${ }^{16} \mathrm{O}$ não planejamento ou desejo da gestação entre os casais, neste estudo, foi considerado relevante. Esses dados manifestam uma inabilidade ou dificuldades no uso de métodos contraceptivos, uma vez que parcela expressiva das mulheres fazia anticoncepção quando engravidou.

Considera-se gravidez não planejada toda e qualquer gestação que não foi programada pelos genitores, podendo ocorrer de forma indesejada, quando se contrapõe às expectativas destes, ou inoportuna, quando ocorre em um período não favorável. ${ }^{15}$ A gravidez não planejada deve ser considerada um problema de saúde pública. Estima-se que todos os anos 80 milhões de mulheres no mundo concebem uma gestação não planejada, e que 60\% delas abortam, agravando os índices de morbimortalidade perinatal. ${ }^{16}$

Apesar da evolução dos métodos contraceptivos ao longo do tempo, assim como o acesso à informação e ao serviço de saúde, nenhum contraceptivo pode ser considerado ideal, devido a suas limitações técnicas e biológicas, no entanto, o seu maior obstáculo é a transposição da barreira cultural. ${ }^{17}$ Nesse sentindo, há ocorrência desse fenômeno no presente estudo. Mulheres com filhos buscaram mais frequentemente pelo serviço de planejamento reprodutivo e uso do contraceptivo. 
Contudo, a literatura mostra que esta procura ocorre não para planejar o número de filhos desejado ou para ter intervalo adequado entre as gestações, mas para cessar a fertilidade. ${ }^{6}$

Da mesma maneira, neste estudo as mulheres que foram submetidas a cesárea anterior não praticavam anticoncepção, estando propensas à esterilização como método contraceptivo. Em contextos com desigualdade social acentuada, a vivência da sexualidade muitas vezes se faz restrita à concepção biológica, estando desvinculada do ato de prazer. Novamente, essas mulheres apresentam menos controle sobre seu corpo, sua capacidade de reprodução e fertilidade, repetindo a procura por medidas de controle da prole por meio da laqueadura. ${ }^{18}$

Embora haja influência positiva no uso de contraceptivos, o serviço público de saúde mostrou-se limitado à oferta de métodos hormonais. Estes são os mais utilizados em todo o mundo, por serem reversíveis, eficientes e disponíveis. No entanto, devido à sua ingestão ser diária, é necessário um uso contínuo e prolongado, tornando-se mais frequente o esquecimento, o que eleva sua taxa de falha. Há também uma relação direta entre o menor nível socioeconômico e a prevalência do consumo de pílulas. ${ }^{19}$ Segundo a pesquisa mundial de planejamento familiar, em 2013, 63\% das mulheres casadas, com idade entre 15 a 49 anos, usavam algum método para o planejamento reprodutivo. Dessas, 57\% utilizavam métodos considerados modernos, que incluem pílulas, DIU, injetáveis, preservativos e esterilização. ${ }^{20}$

No primeiro ano após o parto, verificaram-se tais características na população estudada: mulheres com parceiro, alta concentração do uso métodos hormonais e prevalência de baixa renda. Portanto, para que esse grupo de mulheres exerça sua escolha livre e informada de anticoncepção, é necessário ampliar o acesso aos demais tipos de contraceptivos, empoderar o autoconhecimento do seu corpo e atentar para a vulnerabilidade de mulheres com menor renda. ${ }^{7}$

A assistência à saúde sexual e reprodutiva vem sendo uma das áreas de atuação prioritária da atenção primária, tendo por princípio a garantia dos direitos sexuais e reprodutivos, baseada na Política Nacional de Assistência Integral à Saúde da Mulher e na Política Nacional de 
Planejamento reprodutivo e a vulnerabilidade após o parto: uma coorte do sul do Brasil | 14

Direitos Sexuais e Reprodutivos. ${ }^{21}$ Sendo assim, a APS deveria estar preparada para desenvolver três principais atividades no âmbito de saúde reprodutiva, sendo o aconselhamento, a educação e a clínica. No entanto, as ações se limitam à oferta e distribuição de contraceptivos, com ênfase no ciclo gravídico-puerperal, desviando seu foco da atenção a mulheres que não possuam antecedentes gestacionais, não sejam sexualmente ativas ou, ainda, que sofram com infertilidade. ${ }^{6}$

A consulta puerperal é uma das estratégias de cuidados da APS, principalmente na primeira semana após o parto, pois permite a construção de vínculo com a usuária, proporciona acolhimento e promove atendimento preventivo, holístico e resolutivo frente aos problemas que podem surgir. Contudo, as ações prestadas nesse período estão deficientes de orientações relacionadas ao cuidado integral da mulher, tendo como foco a assistência ao recém-nascido. Portanto, a consulta realizada nesse momento não garante a autoconfiança necessária para que a puérpera desempenhe o autocuidado com excelência. ${ }^{22}$

No estudo, verificou-se que a busca do cuidado pela usuária foi relevante, pois a procura do serviço para planejamento reprodutivo no puerpério remoto resultou na inserção das mulheres neste programa. Ressalta-se, no entanto, que várias puérperas procuraram e foram atendidas pelo serviço de ginecologia, mas não foram inseridas no PPR, reafirmando a fragmentação do cuidado e olhar restrito para a mulher que se encontra no pós-parto.

Esse diagnóstico é contrário à proposta de assistência à saúde na atenção primária e demonstra que o cuidado especializado ainda está centralizando na figura do médico, em detrimento da multiprofissionalidade. A enfermagem, por sua vez, perdeu ou deixou de ocupar um espaço que lhe é garantido, de realizar consultas no acompanhamento do planejamento reprodutivo e prescrição de cuidados e tratamento, mediante estabelecimentos de protocolos. ${ }^{23-24}$

Diante desse fato, verificou-se que a atenção secundária exerceu papel crucial na inclusão de mulheres no PPR, se fazendo em mais uma alternativa para suprir as necessidades da APS. 
15| Canario MASS, Gonçalves MF, Teixeira EMB, Silva AFAQS, Ferrari RAP, Pelloso SM, Cardelli AAM

A atuação do(a) enfermeiro(a) foi fundamental para o resgate das mulheres, nos primeiros dias que sucedem o parto, e sua inserção no PPR, confirmando a sua eficaz participação no puerpério precoce e ação na rede de saúde primária e secundária. O profissional de saúde é visto como agente de poder pela usuária, e é por meio de práticas efetivas em saúde que ela estará fortalecida para escolhas adequadas de anticoncepção e sua prática. ${ }^{25}$

Também foi constatada a evasão das mulheres ao longo do tempo, à medida que deixavam de utilizar os métodos contraceptivos ofertados no serviço público e passavam a obtêlos no setor privado. Essa situação remete à precária captação das puérperas ao longo do primeiro ano, com significativa diminuição da realização da revisão puerperal remota e tardia em comparação à precoce. Um estudo realizado com puérperas em Minas Gerais verificou situação semelhante, na qual 40,2\% das puérperas pretendiam obter o método escolhido em farmácias. ${ }^{26}$

As lacunas deixadas pelo serviço público no Brasil em relação a atenção à saúde reprodutiva denotam a restrição na distribuição dos contraceptivos, pouco envolvimento da equipe e a baixa adesão feminina às atividades propostas. Destaca-se que esses fatores são agravados quando o serviço de saúde desconsidera a influência do gênero na vivência feminina quanto à contracepção. ${ }^{27}$

A fragilidade do acompanhamento ao planejamento reprodutivo pelo serviço público de saúde foi reforçada quando se verificou reincidência de gravidez em algumas mulheres no puerpério tardio e remoto, confirmando o risco de partos prematuros em intervalos curtos de gestações. O principal motivo relatado para não fazer uso de contraceptivo foi a opção de não evitar uma nova gestação. Esta situação faz emergir aspectos que envolvem as relações de gênero, crenças e atitude em saúde e, neste paradigma, a concepção do papel feminino, cultural e socialmente construído, porém baseado na capacidade reprodutora.

A maternidade é vista culturalmente como algo inerente à mulher, instintiva. Também a aptidão para gestar é atribuída historicamente como valor feminino dentro da família. ${ }^{28}$ Acredita-se que, no contexto social brasileiro, a ocorrência de uma gestação não seja suficiente para estimular a adesão ao 
Planejamento reprodutivo e a vulnerabilidade após o parto: uma coorte do sul do Brasil | 16

planejamento reprodutivo, o qual só ocorre de forma significativa após repetição consecutiva da gravidez. A partir disso é que se dá uma busca desesperada pelo controle de natalidade. ${ }^{29}$

A gravidez é decorrente da relação homem e mulher, assim, espera-se que a anticoncepção seja uma decisão de ambos, igualmente envolvidos nesse processo. No entanto, muitas mulheres se encontram em um contexto de pouca ou nenhuma participação do parceiro, sendo a busca contraceptiva uma jornada solitária. Neste sentindo, o serviço de saúde deve se organizar para promover um programa de planejamento reprodutivo que viabilize o respeito à autonomia da mulher, traduzido pelo resgate do cuidado integral ao corpo feminino, seu caráter biológico, emocional e social, e para exercício da paternidade responsável. O perfil itinerante das mulheres abordadas na pesquisa resultou na dificuldade da coleta de dados para a terceira e quarta etapas do estudo, reduzindo a amostra ao longo do primeiro ano após o parto.

\section{Conclusão}

O seguimento da usuária, no período do primeiro ano após o parto, demonstrou que consulta ambulatorial puerperal realizada no serviço secundário e consulta puerperal precoce realizada na atenção primária foram positivas para inscrição da mulher no planejamento reprodutivo, com predomínio do profissional enfermeiro para realização da consulta. No entanto, as fragilidades identificadas, como descontinuidade no acompanhamento da revisão no puerpério remoto, assistência e orientações voltadas principalmente para saúde da criança, além da baixa inscrição das mulheres no PPR durante todo o período do primeiro ano após o parto, mostraram-se fortemente influentes na não adesão ao cuidado reprodutivo por meio do uso de contraceptivos.

Embora o pós-parto seja um período de vulnerabilidade feminina, constatou-se, no estudo, restrição na aplicação das políticas de saúde sexual e reprodutiva, sendo a principal atuação a oferta e distribuição de contraceptivos hormonais. É emergente o resgate e a aplicação do 
conceito de planejamento reprodutivo, já que as demandas desse período necessitam de ações efetivas do serviço de saúde e comprometimento dos profissionais de saúde.

Por meio deste estudo é possível reafirmar a vulnerabilidade da mulher após o parto, considerando os aspectos reprodutivos. Essa é uma população em que as estratégias políticas e programas de saúde, como assistência após o parto, que inclui a revisão puerperal com orientação ao planejamento reprodutivo, não produz o resultado apropriado.

Assim, é nítido que a atuação do profissional enfermeiro, quando realizada de forma adequada, é capaz de transformar uma realidade social e permitir o acesso da usuária àquilo que lhe é de direito, com informação, prevenção e prestação de serviço.

\section{Referências}

1. Andrade RD, Santos JS, Maia MAC, Mello DF. Fatores relacionados à saúde da mulher no puerpério e repercussões na saúde da criança. Esc Anna Nery. 2015;19(1):181-6. doi: 10.5935/1414-8145.20150025

2. Ministério da Saúde (BR), Secretaria de Atenção à Saúde, Departamento de Atenção Básica. Saúde sexual e saúde reprodutiva. Brasília (DF): Ministério da Saúde; 2013.

3. Paiva CCN, Caetano R. Avaliação de implantação das ações de saúde sexual e reprodutiva na Atenção Primária: revisão de escopo. Esc Anna Nery. 2020;24(1). doi: https://doi.org/10.1590/2177-9465-ean-2019-0142

4. Gonçalves CS, Cesar JA, Marmitt LP, Gonçalves CV. Frequency and associated factors with failure to perform the puerperal consultation in a cohort study. Rev Bras Saúde Mater Infant. 2019;19(1):63-70. doi: $10.1590 / 1806-93042019000100004$

5. Paiva CCN, Villar ASE, Silva MDB, Souza NAS, Lemos A. Themes addressed in educational groups of sexual and reproductive health: an integrative review. Rev Pesq Cuid Fundam. 2016;8(3):4872-81. doi: 10.9789/2175-5361.2016.v8i3.4872-4881

6. Moura LNB, Gomes KRO. Planejamento familiar: uso dos serviços de saúde por jovens com experiência de gravidez. Ciênc Saúde Colet. 2014;19(3):853-63. doi: 10.1590/1413-81232014193.10902013

7. Nações Unidas no Brasil (ONUBR) .Glossário de termos do Objetivo de Desenvolvimento Sustentável 5: alcançar a igualdade de gênero e empoderar todas as mulheres e meninas; 2016.

8. Tran NT, Yameogo WMM, Langwana F, Kouanda S, Thieba B, Mashinda D, et al. Birth spacing and informed family planning choices after childbirth in Burkina Faso and the Democratic Republic of Congo: participatory action research to design and evaluate a decision-making tool for providers and 
Planejamento reprodutivo e a vulnerabilidade após o parto: uma coorte do sul do Brasil | 18

their clients. Patient Educ Couns. 2018 Oct;101(10):1871-5. doi: 10.1016/j.pec.2018.05.004

9. Prefeitura do Município de Londrina, Secretaria Municipal de Saúde. Plano Municipal de Saúde 2018-2021 [Internet]. Londrina (PR): Prefeitura do Município de Londrina; 2017 [acesso em 2020 jul 22]. Disponível em: http://www1.londrina.pr.gov.br/dados/images/stories/Storage/sec_saude/Plano\%20Municial/plano_municipal_2018_2021.pdf.

10. Araujo AP, Oliveira CA, Germano EL, Santos TAS. O pluralismo familiar e a liberdade de constituição de uma comunhão da vida familiar [Internet]. Mostra Trab Academ FAF. 2015 [acesso em 2020 jul 22];3(2015). Disponível em: http://ienomat.com.br/revistas/mtac/index.php/mtac/article/view/69

11. Darsie C, Rocha CF, Silva VC, Koetz APM, Gama A, Dias S. Saúde sexual e reprodutiva e planejamento familiar no contexto de imigrantes brasileiras e africanas que vivem em Portugal. Sanare (Sobral) [Internet]. 2014 [acesso em 2020 jul 01];13(2). Disponível em: https://sanare.emnuvens.com.br/sanare/article/view/567/301

12. Evangelista CB, Barbieri M, Silva PLN. Gravidez não planejada e fatores associados à participação em programa de planejamento familiar. Rev Pesq Cuid Fundam [Internet]. 2015 [acesso em 2020 jul 13];7(2):2464-74. Disponível em: https://www.redalyc.org/articulo.oa?id=505750946023

13. Bawah AA, P Asuming, Achana SF, Kanmiki EW, Awoonor-Williams JK, Phillips JF. Intenções de uso de contraceptivos e necessidade não atendida de planejamento familiar entre mulheres em idade reprodutiva na região do Oriente Médio de Gana. Reprod Health. 2019;16(1):26. doi: 10.1186/s12978-019-0693-x

14. Callegari LS, Aiken ARA, Dehlendorf C, Borrero S. Planejamento da vida reprodutiva e assistência centrada no paciente: as inconsistências podem ser reconciliadas? Matern Child Health J. 2019 Jul;23(7):869-70. doi: 10.1007/s10995-019-02734-3

15. Castro S, Rocha FCG, Góis EG, Sousa DJ, Góis EG, Carvalho ML. Métodos contraceptivos utilizados no planejamento familiar em mulheres de baixa renda em São Luís - MA. Rev Interdiscip [Internet]. 2015 [acesso em 2020 jul 01];8(1):29-136. Disponível em: https://revistainterdisciplinar.uninovafapi.edu.br/index.php/revinter/article/view/575/pdf_191.

16. Cruz R, Morais ACB, Pinto SL, Amorim LTCG, Sampaio KJAJ. Participação masculina no planejamento familiar: o que pensam as mulheres? Cogitare Enferm. 2014;19(4). doi: 10.5380/ce.v19i4.37086

17. Conceição SP, Fernandes RAQ. Influência da gravidez não planejada no tempo de aleitamento materno. Esc Anna Nery. 2015;19(4):600-5. doi: 10.5935/1414-8145.20150080

18. Dourado I, MacCarthy S, Reddy M, Calazans G, Gruskin S. Revisitando o uso do preservativo no Brasil. Rev Bras Epidemiol. 2015;18(1):63-88. doi: 10.1590/1809-4503201500050006

19. Tintori, JA, Cruz LF, Simon CP, Santos DB. Conhecimento, utilização e orientação sobre métodos contraceptivos com mulheres. Rev Ed Popul [Internet]. 2016 [acesso em 2020 jul 12];15(1):169-79. 
19| Canario MASS, Gonçalves MF, Teixeira EMB, Silva AFAQS, Ferrari RAP, Pelloso SM, Cardelli AAM

Disponível em: http://www.seer.ufu.br/index.php/reveducpop/article/view/35691/18801

20. Population Reference Bureau (PRB). Family planning worldwide - 2013 data sheet [Internet]. Washington (DC): Population Reference Bureau; 2013 [cited 2020 Jul 03]. Available from: https://assets.prb.org/pdf13/family-planning-2013-datasheet_eng.pdf

21. Tamboril ACR, Alcântara JAS, Áfio ACE, Sousa CSP, Rebouças LN, Moura ERF, et al. Nursing diagnosis "Deficient Knowledge" in users of combined oral contraceptive. Rev Eletrônica Enferm [Internet]. 2015 [cited 2020 Jul 13];17(4). Available from: https://revistas.ufg.br/fen/article/view/31786/20674

22. Fonseca ACN, Gomes AT, Barreto JG. Distribuição de anticoncepcionais em uma farmácia básica no município de São José do Calçado - ES. Acta Biomed Bras [Internet]. 2015 [acesso em 2020 jun 14];6(1):1020. Disponível em: http://www.actabiomedica.com.br/index.php/acta/article/view/107/80

23. Luiz MS, Nakano AR, Bonan C. Planejamento reprodutivo na clínica da família de um Teias: condições facilitadoras e limites à assistência. Saúde Debate. 2015;39(106):671-82. doi: 10.1590/0103110420151060003009

24. Medeiros LS, Costa ACM. Período puerperal: a importância da visita domiciliar para enfermeiros da Atenção Primária à Saúde. Rev Rene (Online). 2016;17(1):112-9. doi: 10.15253/2175-6783.2016000100015

25. Pereira MC, Gradim CVC. Consulta puerperal: a visão do enfermeiro e da puérpera. Ciênc Cuid Saúde [Internet]. 2014 [acesso em 2020 jun 22];13(1):35-42. Disponível em: http://www.periodicos.uem.br/ojs/index.php/CiencCuidSaude/article/view/19572/pdf_110

26. Mazzo MHSN, Brito R. Instrumento para consulta de enfermagem à puérpera na atenção básica. Rev Bras Enferm. 2016;69(2):316-25. doi: 10.1590/0034-7167.2016690215I

27. Camillo BS, Nietsche EA, Salbego C, Cassenote LG, Dal-Osto DS, Böck A. Ações de educação em saúde na atenção primária às gestantes e puérperas: revisão integrativa. Rev Enferm UFPE On Line. 2016 [cited 2020 Jul 12];10(6):4894-901. Available from: https://periodicos.ufpe.br/revistas/revistaenfermagem/article/view/11270

28. Medeiros TFR, Santos SMP, Xavier AG, Gonçalves RL, Mariz SR, Sousa FLP. Vivência de mulheres sobre contracepção na perspectiva de gênero. Rev Gaúcha Enferm. 2016;37(2):e57350. doi: 10.1590/19831447.2016.02.57350

29. Assunção PN, Bagattini R, Cordeiro F. Métodos contraceptivos utilizados por mulheres residentes na região sul da cidade de São Paulo. Um tipo de estudo cap; conhecimento, atitude e prática. Atas Saúde Ambient [Internet]. 2017 [acesso em 2020 jun 20];5(1):157-71. Disponível em: http://revistaseletronicas.fmu.br/index.php/ASA/article/view/1617

Editora Científica Chefe: Cristiane cardo de Paula

Editora Científica: Tânia Solange Bosi de Souza Magnago 
Planejamento reprodutivo e a vulnerabilidade após o parto: uma coorte do sul do Brasil | 20

Fomento / Agradecimento: CAPES/ Fundação Araucária

\section{Autor correspondente}

Márcia Aparecida dos Santos Silva Canario

E-mail: marcia.s.s.canario@gmail.com

Endereço: rua Mario Oncken, $105 \mathrm{Jd}$. Américas

CEP: 86076090

\section{Contribuições de autoria}

\section{1 - Márcia Aparecida dos Santos Silva Canario}

Concepção ou desenho do estudo/pesquisa, análise e/ou interpretação dos dados, revisão final com participação crítica e intelectual no manuscrito.

\section{2 - Mariana Faria Gonçalves}

Análise e/ou interpretação dos dados, revisão final com participação crítica e intelectual no manuscrito.

\section{3 - Érica Mairene Bocate Teixeira}

Análise e/ou interpretação dos dados, revisão final com participação crítica e intelectual no manuscrito.

\section{4 - Áurea Fabrícia Amancio Quirino Silva}

Concepção ou desenho do estudo/pesquisa, análise e/ou interpretação dos dados

\section{5 - Rosângela Aparecida Pimenta Ferrari}

Revisão final com participação crítica e intelectual no manuscrito.

\section{6 - Sandra Marisa Pelloso}

Revisão final com participação crítica e intelectual no manuscrito.

\section{7 - Alexandrina Aparecida Maciel Cardelli}

Análise e/ou interpretação dos dados, revisão final com participação crítica e intelectual no manuscrito.

\section{Como citar este artigo}

Canario MASS, Gonçalves MF, Teixeira EMB, Silva AFAQS, Ferrari RAP, Pelloso SM, Cardelli AAM. Planejamento reprodutivo e a vulnerabilidade após o parto: uma coorte do sul do Brasil. Rev. Enferm. UFSM. 2020 [Acesso em: Anos Mês Dia]; vol.10 e: 1-20. DOI:https://doi.org/10.5902/2179769240659 